\title{
MIMO Gaussian X Channel: Noisy Interference Regime
}

\author{
Praneeth Kumar V. and Srikrishna Bhashyam, Senior Member, IEEE
}

\begin{abstract}
The two-user multiple-input multiple-output (MIMO) Gaussian X channel (XC) consists of two transmitters and two receivers, with each transmitter having an independent message to each receiver. The sum capacity of the two-user MIMO Gaussian XC is determined in a noisy interference regime. This sum capacity is achieved by using Gaussian codebooks for the messages on both the direct links (or both the cross links) and treating the interference from the cross links (or direct links) as noise.
\end{abstract}

Index Terms-Interference channel, $\mathrm{X}$ channel, sum capacity, MIMO.

\section{INTRODUCTION}

$\mathbf{T}$ HE two-user Interference Channel (IC) consists of two interfering transmitter-receiver pairs where each transmitter has a message to its own receiver. The sum capacity of the two-user scalar Gaussian IC in the noisy interference regime was determined in [1]. In this regime, the cross links are weak enough that using Gaussian codebooks and treating the interference as noise is sum-capacity optimal. A similar noisy interference regime was obtained for the two-user multipleinput multiple-output (MIMO) Gaussian IC in [2].

The two-user X channel (XC) is a generalization of the twouser IC where both transmitters have independent messages for both receivers. Thus, there are four messages instead of two messages. In [3], it was shown that in the noisy interference regime the sum capacity of the scalar Gaussian XC is the same as that of the scalar Gaussian IC. Therefore, treating interference as noise and transmitting only two messages is optimal even for the scalar Gaussian XC in the noisy interference regime [3]. In this work, we first obtain a similar result for the MIMO Gaussian XC (see Fig. 1). We show that in the noisy interference regime identified in [2] the sum capacity of the MIMO Gaussian XC is the same as that of the MIMO Gaussian IC. This is proved by appropriately using the genie chosen in [3] along with the proof technique used in [2] for the MIMO Gaussian IC. Then, the results for the multiple-input singleoutput (MISO) Gaussian IC and single-input multiple-output (SIMO) Gaussian IC in [2] are also extended to the MISO Gaussian XC and SIMO Gaussian XC, respectively. Finally, the sum capacity and the corresponding optimal power allocation are determined for the symmetric diagonal MIMO Gaussian XC in the noisy interference regime.

It should be noted that a single noisy interference region for the IC corresponds to multiple noisy interference regions for

Manuscript received March 28, 2014; accepted June 4, 2014. Date of publication June 12, 2014; date of current version August 8, 2014. This work was done at the Department of Electrical Engineering, Indian Institute of Technology Madras. The associate editor coordinating the review of this paper and approving it for publication was M. Wigger.

Praneeth Kumar V. is with the ISRO, Bangalore 560094, India.

$\mathrm{S}$. Bhashyam is with the Department of Electrical Engineering, Indian Institute of Technology Madras, Chennai 600036, India.

Color versions of one or more of the figures in this paper are available online at http://ieeexplore.ieee.org.

Digital Object Identifier 10.1109/LCOMM.2014.2329862

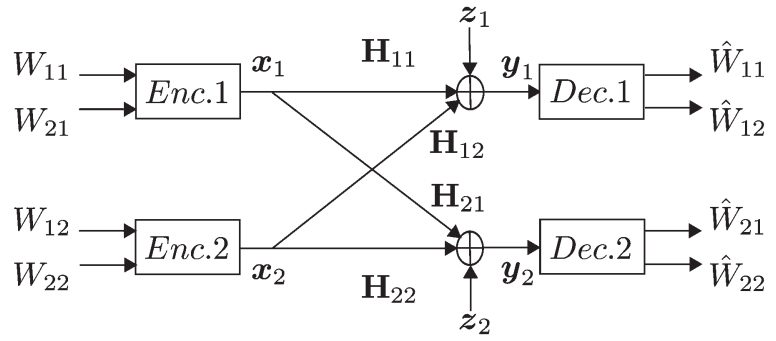

Fig. 1. Two user MIMO Gaussian XC.

the XC. Two such cases are where: (1) the cross links are weak and the interference from the cross links can be treated as noise while decoding the direct messages, or (2) the direct links are weak and the interference from the direct links can be treated as noise while decoding the cross messages. In the symmetric diagonal MIMO XC, each of the parallel XCs could be in any of the above two noisy interference regimes for the overall MIMO $\mathrm{XC}$ to be in a noisy interference regime.

\section{SYSTEM MODEL}

We consider the two user MIMO Gaussian XC in Fig. 1, which is same as the MIMO Gaussian IC except that each transmitter has separate independent messages for both receivers. The MIMO Gaussian XC is described by the following equations:

$$
\begin{aligned}
& \boldsymbol{y}_{1}=\mathbf{H}_{11} \boldsymbol{x}_{1}+\mathbf{H}_{12} \boldsymbol{x}_{2}+\boldsymbol{z}_{1}, \\
& \boldsymbol{y}_{2}=\mathbf{H}_{21} \boldsymbol{x}_{1}+\mathbf{H}_{22} \boldsymbol{x}_{2}+\boldsymbol{z}_{2},
\end{aligned}
$$

where $\boldsymbol{x}_{i}$ is a $t_{i} \times 1$ vector, $\boldsymbol{y}_{i}, \boldsymbol{z}_{i}$ are $r_{i} \times 1$ vectors, $\mathbf{H}_{i j}$ is $r_{i} \times t_{j}$ channel matrix and $t_{i}, r_{j}$ are the number of antennas at transmitter $i$, receiver $j$ respectively. Noise vector $\boldsymbol{z}_{i} \sim \mathcal{N}\left(\underline{0}, I_{r_{i} \times r_{i}}\right)$ and is i.i.d. across time. The average power constraint on the $i$ th transmitter over an $n$ symbol duration is

$$
\frac{1}{n} \sum_{k=1}^{n} E\left[\boldsymbol{x}_{i k} \boldsymbol{x}_{i k}^{T}\right] \in \mathcal{Q}_{i},
$$

where $\mathcal{Q}_{i}=\left\{\mathbf{Q}_{i}: \mathbf{Q}_{i} \succeq 0, \operatorname{tr}\left(\mathbf{Q}_{i}\right) \leq P_{i}\right\}$.

Rate $R_{i j}$ is the rate of reliable transmission from transmitter $j$ to receiver $i$. An achievable rate over the MIMO Gaussian $\mathrm{XC}$ is characterized by the rate 4 -tuple $\left(R_{11}, R_{21}, R_{12}, R_{22}\right)$. The capacity region is defined as the closure of all achievable rate tuples, and the sum capacity is the maximum achievable sum rate $R_{11}+R_{21}+R_{12}+R_{22}$.

\section{Sum CAPACITy In THE NOISy Interference Regime}

The MIMO Gaussian XC is defined by the parameters $\left\{\mathbf{H}_{11}\right.$, $\left.\mathbf{H}_{21}, \mathbf{H}_{12}, \mathbf{H}_{22}\right\}$ and the power constraints $\left\{P_{1}, P_{2}\right\}$. Let us denote the channel in Fig. 1 by $\operatorname{GXC}\left(\mathbf{H}_{11}, \mathbf{H}_{21}, \mathbf{H}_{12}\right.$, $\left.\mathbf{H}_{22}, P_{1}, P_{2}\right)$.

Definition 1 (Dual Channel): $\mathrm{GXC}\left(\mathbf{H}_{21}, \mathbf{H}_{11}, \mathbf{H}_{22}, \mathbf{H}_{12}\right.$, $\left.P_{1}, P_{2}\right)$ is the dual channel of $\operatorname{GXC}\left(\mathbf{H}_{11}, \mathbf{H}_{21}, \mathbf{H}_{12}, \mathbf{H}_{22}\right.$, 
$\left.P_{1}, P_{2}\right)$ and is described by the following equations:

$$
\begin{aligned}
& \boldsymbol{y}_{1}^{\prime}=\mathbf{H}_{21} \boldsymbol{x}_{1}^{\prime}+\mathbf{H}_{22} \boldsymbol{x}_{2}^{\prime}+\boldsymbol{z}_{1}^{\prime} \\
& \boldsymbol{y}_{2}^{\prime}=\mathbf{H}_{11} \boldsymbol{x}_{1}^{\prime}+\mathbf{H}_{12} \boldsymbol{x}_{2}^{\prime}+\boldsymbol{z}_{2}^{\prime} .
\end{aligned}
$$

The dual channel can be obtained from the original channel by re-indexing receiver 1 in the original channel as receiver 2 in the dual channel, and receiver 2 in the original channel as receiver 1 in the dual channel. Messages $W_{11}^{\prime}, W_{21}^{\prime}, W_{12}^{\prime}, W_{22}^{\prime}$ in the dual channel are the same as messages $W_{21}, W_{11}, W_{22}, W_{12}$ respectively, for the original channel.

Remark 1: $\left(R_{11}, R_{21}, R_{12}, R_{22}\right)$ is achievable in the channel $\operatorname{GXC}\left(\mathbf{H}_{11}, \mathbf{H}_{21}, \mathbf{H}_{12}, \mathbf{H}_{22}, P_{1}, P_{2}\right)$ if and only if $\left(R_{11}^{\prime}, R_{21}^{\prime}, R_{12}^{\prime}, R_{22}^{\prime}\right)=\left(R_{21}, R_{11}, R_{22}, R_{12}\right)$ is achievable in the channel $\operatorname{GXC}\left(\mathbf{H}_{21}, \mathbf{H}_{11}, \mathbf{H}_{22}, \mathbf{H}_{12}, P_{1}, P_{2}\right)$.

Remark 2: $\operatorname{GXC}\left(\mathbf{H}_{11}, \mathbf{H}_{21}, \mathbf{H}_{12}, \mathbf{H}_{22}, P_{1}, P_{2}\right)$ and the dual channel $\operatorname{GXC}\left(\mathbf{H}_{21}, \mathbf{H}_{11}, \mathbf{H}_{22}, \mathbf{H}_{12}, P_{1}, P_{2}\right)$ have the same sum capacity.

\section{A. TIN Scheme}

In the Treating Interference as Noise (TIN) scheme for $\operatorname{GXC}\left(\mathbf{H}_{11}, \mathbf{H}_{21}, \mathbf{H}_{12}, \mathbf{H}_{22}, P_{1}, P_{2}\right)$, only the two direct messages $W_{11}, W_{22}$ are sent using Gaussian codebooks and the interference is treated as noise at both receivers. Let $R_{T I N}^{X}$ be the achievable sum rate by treating interference as noise for given $\mathbf{Q}_{1}, \mathbf{Q}_{2}$ and

$$
\left(\mathbf{Q}_{1}^{*}, \mathbf{Q}_{2}^{*}\right)=\underset{\mathbf{Q}_{i} \in \mathcal{Q}_{i}, i \in\{1,2\}}{\arg \max } R_{T I N}^{X}\left(\mathbf{Q}_{1}, \mathbf{Q}_{2}\right) .
$$

Another TIN scheme would be to send only the two cross messages $W_{12}, W_{21}$ using Gaussian codebooks and treat interference as noise at the receivers. This is the same as using the TIN scheme with direct messages for the dual channel $\operatorname{GXC}\left(\mathbf{H}_{21}, \mathbf{H}_{11}, \mathbf{H}_{22}, \mathbf{H}_{12}, P_{1}, P_{2}\right)$.

\section{B. Noisy Interference Regime}

First, we determine the noisy interference regime where the TIN scheme with direct messages is sum capacity optimal. The noisy interference regime where the TIN scheme with cross messages is sum capacity optimal follows directly by applying the result for direct messages to the dual channel.

Theorem 1: If there exist matrices $\mathbf{A}_{1}, \mathbf{A}_{2}, \boldsymbol{\Sigma}_{1} \succ 0, \boldsymbol{\Sigma}_{2} \succ 0$, full rank matrices $\left(\mathbf{Q}_{1}^{*}, \mathbf{Q}_{2}^{*}\right)$ that solve problem (3), and they satisfy the following conditions:

$$
\begin{aligned}
& \boldsymbol{\Sigma}_{1} \preceq I-\mathbf{A}_{2} \boldsymbol{\Sigma}_{2}^{-1} \mathbf{A}_{2}^{T} \\
& \boldsymbol{\Sigma}_{2} \preceq I-\mathbf{A}_{1} \boldsymbol{\Sigma}_{1}^{-1} \mathbf{A}_{1}^{T} \\
& \mathbf{A}_{1}^{T}\left(\mathbf{H}_{12} \mathbf{Q}_{2}^{*} \mathbf{H}_{12}^{T}\right)^{-1} \mathbf{H}_{11}-\mathbf{H}_{21}=0 \\
& \mathbf{A}_{2}^{T}\left(\mathbf{H}_{21} \mathbf{Q}_{1}^{*} \mathbf{H}_{21}^{T}\right)^{-1} \mathbf{H}_{22}-\mathbf{H}_{12}=0,
\end{aligned}
$$

the sum capacity of the MIMO Gaussian XC $\operatorname{GXC}\left(\mathbf{H}_{11}, \mathbf{H}_{21}\right.$, $\left.\mathbf{H}_{12}, \mathbf{H}_{22}, P_{1}, P_{2}\right)$ is achieved by the TIN scheme with direct messages only, and is given by:

$$
\begin{aligned}
C_{\text {sum }}^{X}= & R_{T I N}^{X}\left(\mathbf{Q}_{1}^{*}, \mathbf{Q}_{2}^{*}\right) \\
= & \max _{\mathbf{Q}_{i} \in \mathcal{Q}_{i}, i \in\{1,2\}} \frac{1}{2} \log \mid I+\mathbf{H}_{11} \mathbf{Q}_{1} \mathbf{H}_{11}^{T} \\
& \quad \times\left(I+\mathbf{H}_{12} \mathbf{Q}_{2} \mathbf{H}_{12}^{T}\right)^{-1} \mid \\
& +\frac{1}{2} \log \left|I+\mathbf{H}_{22} \mathbf{Q}_{2} \mathbf{H}_{22}^{T}\left(I+\mathbf{H}_{21} \mathbf{Q}_{1} \mathbf{H}_{21}^{T}\right)^{-1}\right| .
\end{aligned}
$$

Proof: Consider the genie-aided channel with a genie providing side information $s_{1}, W_{21}$ to receiver 1 and $s_{2}, W_{12}$ to receiver 2 , where

$$
\begin{aligned}
\boldsymbol{s}_{1} & =\mathbf{H}_{21} \boldsymbol{x}_{1}+\boldsymbol{w}_{1} \\
\boldsymbol{s}_{2} & =\mathbf{H}_{12} \boldsymbol{x}_{2}+\boldsymbol{w}_{2}
\end{aligned}
$$

and $\left[\begin{array}{c}\boldsymbol{z}_{i} \\ \boldsymbol{w}_{i}\end{array}\right] \sim \mathcal{N}\left(\underline{0},\left[\begin{array}{cc}I & \mathbf{A}_{i} \\ \mathbf{A}_{i}^{T} & \boldsymbol{\Sigma}_{i}\end{array}\right]\right)$.

Here, $s_{1}$ and $s_{2}$ are chosen as for the MIMO-IC in [2], and $W_{21}$ and $W_{12}$ are provided to receivers 1 and 2 respectively as in [3] for the SISO XC. Using Fano's inequality, we have

$$
\begin{aligned}
& h\left(R_{11}+R_{12}-\epsilon\right) \\
& \leq I\left(W_{11}, W_{12} ; \boldsymbol{y}_{1}^{n}, \boldsymbol{s}_{1}^{n}, W_{21}\right) \\
&= I\left(W_{11}, W_{12} ; \boldsymbol{y}_{1}^{n}, \boldsymbol{s}_{1}^{n} \mid W_{21}\right)+I\left(W_{11}, W_{12} ; W_{21}\right) \\
& \stackrel{(a)}{=}
\end{aligned}
$$

where step (a) follows from the independence of the messages, step (b) follows from the deterministic encoding of $W_{11}, W_{21}$ to $\boldsymbol{x}_{1}^{n}$, step (c) follows from the fact that conditioning reduces the entropy, and step (d) is because the Gaussian distribution maximizes the conditional entropy [2, Lemma 7].

Similarly, we have

$$
\begin{array}{r}
n\left(R_{21}+R_{22}-\epsilon\right) \leq h\left(\boldsymbol{s}_{2}^{n} \mid W_{12}\right)-n h\left(\boldsymbol{w}_{2}\right)+n h\left(\boldsymbol{y}_{2 G} \mid \boldsymbol{s}_{2 G}\right) \\
-h\left(\mathbf{H}_{21} \boldsymbol{x}_{1}^{n}+\boldsymbol{z}_{2}^{n} \mid \boldsymbol{w}_{2}^{n}, W_{21}\right) . \quad \text { (19) }
\end{array}
$$

Adding (18) and (19), we have

$$
\begin{aligned}
& n\left(C_{\text {sum }}^{G A-X}-2 \epsilon\right) \\
& \leq {\left[h\left(\boldsymbol{s}_{1}^{n} \mid W_{21}\right)-h\left(\mathbf{H}_{21} \boldsymbol{x}_{1}^{n}+\boldsymbol{z}_{2}^{n} \mid \boldsymbol{w}_{2}^{n}, W_{21}\right)\right] } \\
&+\left[h\left(\boldsymbol{s}_{2}^{n} \mid W_{12}\right)-h\left(\mathbf{H}_{12} \boldsymbol{x}_{2}^{n}+\boldsymbol{z}_{1}^{n} \mid \boldsymbol{w}_{1}^{n}, W_{12}\right)\right] \\
&-n h\left(\boldsymbol{w}_{1}\right)+n h\left(\boldsymbol{y}_{1 G} \mid \boldsymbol{s}_{1 G}\right) \\
&-n h\left(\boldsymbol{w}_{2}\right)+n h\left(\boldsymbol{y}_{2 G} \mid \boldsymbol{s}_{2 G}\right),
\end{aligned}
$$

where $C_{\text {sum }}^{G A-X}$ is the sum capacity of the genie-aided MIMO $\mathrm{XC}$. Now, consider the first term in (20).

$$
\begin{aligned}
& h\left(\boldsymbol{s}_{1}^{n} \mid W_{21}\right)-h\left(\mathbf{H}_{21} \boldsymbol{x}_{1}^{n}+\boldsymbol{z}_{2}^{n} \mid \boldsymbol{w}_{2}^{n}, W_{21}\right) \\
& \quad=h\left(\mathbf{H}_{21} \boldsymbol{x}_{1}^{n}+\boldsymbol{w}_{1}^{n} \mid W_{21}\right)-h\left(\mathbf{H}_{21} \boldsymbol{x}_{1}^{n}+\boldsymbol{z}_{2}^{n} \mid \boldsymbol{w}_{2}^{n}, W_{21}\right) \\
& \quad \stackrel{(e)}{=} h\left(\mathbf{H}_{21} \boldsymbol{x}_{1}^{n}+\boldsymbol{w}_{1}^{n} \mid W_{21}\right)-h\left(\mathbf{H}_{21} \boldsymbol{x}_{1}^{n}+\boldsymbol{v}_{1}^{n} \mid W_{21}\right) \\
& \quad \stackrel{(f)}{=} h\left(\mathbf{H}_{21} \boldsymbol{x}_{1}^{n}+\boldsymbol{w}_{1}^{n} \mid W_{21}\right)-h\left(\mathbf{H}_{21} \boldsymbol{x}_{1}^{n}+\boldsymbol{w}_{1}^{n}+\tilde{\boldsymbol{v}}_{1}^{n} \mid W_{21}\right) \\
& \quad=-I\left(\tilde{\boldsymbol{v}}_{1}^{n} ; \mathbf{H}_{21} \boldsymbol{x}_{1}^{n}+\boldsymbol{w}_{1}^{n}+\tilde{\boldsymbol{v}}_{1}^{n} \mid W_{21}\right) \\
& \quad \stackrel{(g)}{\leq}-I\left(\tilde{\boldsymbol{v}}_{1}^{n} ; \mathbf{H}_{21} \boldsymbol{x}_{1}^{n}+\boldsymbol{w}_{1}^{n}+\tilde{\boldsymbol{v}}_{1}^{n}\right)
\end{aligned}
$$




$$
\begin{aligned}
& \stackrel{(h)}{\leq}-n I\left(\tilde{\boldsymbol{v}}_{1} ; \mathbf{H}_{21} \boldsymbol{x}_{1 G}+\boldsymbol{w}_{1}+\tilde{\boldsymbol{v}}_{1}\right) \\
& =n h\left(\boldsymbol{s}_{1 G}\right)-n h\left(\mathbf{H}_{21} \boldsymbol{x}_{1 G}+\boldsymbol{z}_{2} \mid \boldsymbol{w}_{2}\right),
\end{aligned}
$$

where $\boldsymbol{v}_{1}^{n}$ is the MMSE error in estimating $\boldsymbol{z}_{2}^{n}$ given $\boldsymbol{w}_{2}^{n}$ and $\boldsymbol{v}_{1}^{n} \sim \mathcal{N}\left(\underline{0}, I-\mathbf{A}_{2} \boldsymbol{\Sigma}_{2}^{-1} \mathbf{A}_{2}^{T}\right)$, and step (e) follows from [2, Lemma 9]. In step (f), $\tilde{\boldsymbol{v}}_{1}^{n} \sim \mathcal{N}\left(\underline{0}, I-\mathbf{A}_{2} \boldsymbol{\Sigma}_{2}^{-1} \mathbf{A}_{2}^{T}-\boldsymbol{\Sigma}_{1}\right)$ and is independent of $\boldsymbol{w}_{1}^{n}$. Since the covariance matrix has to be positive semidefinite, we get the condition (4). Step (f) follows since $\boldsymbol{w}_{1}^{n}+\tilde{\boldsymbol{v}}_{1}^{n}$ has the same marginal as $\boldsymbol{v}_{1}^{n}$. Step (g) follows since $\tilde{\boldsymbol{v}}_{1}$ is independent of $W_{21}$ and conditioning reduces entropy. Step (h) follows from the worst case noise result [2, Lemma 8]. Similarly, the second term in (20) can also be simplified and we get condition (5) in the process. Overall, we get

$$
\begin{aligned}
& n\left(C_{\text {sum }}^{G A-X}-2 \epsilon\right) \leq\left[n h\left(\boldsymbol{s}_{1 G}\right)-n h\left(\mathbf{H}_{21} \boldsymbol{x}_{1 G}+\boldsymbol{z}_{2} \mid \boldsymbol{w}_{2}\right)\right] \\
& \quad+\left[n h\left(\boldsymbol{s}_{2 G}\right)-n h\left(\mathbf{H}_{12} \boldsymbol{x}_{2 G}+\boldsymbol{z}_{1} \mid \boldsymbol{w}_{1}\right)\right] \\
& \quad-n h\left(\boldsymbol{w}_{1}\right)+n h\left(\boldsymbol{y}_{1 G} \mid \boldsymbol{s}_{1 G}\right)-n h\left(\boldsymbol{w}_{2}\right)+n h\left(\boldsymbol{y}_{2 G} \mid \boldsymbol{s}_{2 G}\right) \\
& \quad=\sum_{i=1}^{2} n I\left(\boldsymbol{x}_{i G} ; \boldsymbol{y}_{i G}, \boldsymbol{s}_{i G}\right) \triangleq n R_{T I N}^{G A-X}\left(\mathbf{Q}_{1}, \mathbf{Q}_{2}\right) .
\end{aligned}
$$

Therefore, we have

$$
\begin{aligned}
C_{\text {sum }}^{G A-X} & \leq R_{T I N}^{G A-X}\left(\mathbf{Q}_{1}, \mathbf{Q}_{2}\right) \quad \text { for some } \mathbf{Q}_{i} \in \mathcal{Q}_{i} \\
& \leq \max _{\mathbf{Q}_{i} \in \mathcal{Q}_{i}, i \in\{1,2\}} R_{T I N}^{G A-X}\left(\mathbf{Q}_{1}, \mathbf{Q}_{2}\right)
\end{aligned}
$$

resulting in $C_{\text {sum }}^{G A-X}=\max _{\mathbf{Q}_{i} \in \mathcal{Q}_{i}, i \in\{1,2\}} R_{T I N}^{G A-X}\left(\mathbf{Q}_{1}, \mathbf{Q}_{2}\right)$. At this point, for a given $\mathbf{Q}_{1}, \mathbf{Q}_{2}$, the genie-aided outer bound $R_{T I N}^{G A-X}\left(\mathbf{Q}_{1}, \mathbf{Q}_{2}\right)$ is the same as the genie-aided outer bound in [2] for the MIMO-IC. Therefore, the remaining steps that involve proving that the genie does not increase sum capacity, i.e., proving the smart genie conditions (6), (7) and $R_{T I N}^{G A-X}\left(\mathbf{Q}_{1}^{*}, \mathbf{Q}_{2}^{*}\right)=R_{T I N}^{X}\left(\mathbf{Q}_{1}^{*}, \mathbf{Q}_{2}^{*}\right)$, where $\mathbf{Q}_{1}^{*}, \mathbf{Q}_{2}^{*}$ are full rank matrices, are similar to [2, Theorem 1] and are not repeated here.

Theorem 2: If there exist matrices $\mathbf{A}_{1}, \mathbf{A}_{2}, \boldsymbol{\Sigma}_{1} \succ 0, \boldsymbol{\Sigma}_{2} \succ 0$, full rank matrices $\left(\mathbf{Q}_{1}^{*}, \mathbf{Q}_{2}^{*}\right)$ which solve problem (3), and they satisfy the following conditions:

$$
\begin{aligned}
& \boldsymbol{\Sigma}_{1} \preceq I-\mathbf{A}_{2} \boldsymbol{\Sigma}_{2}^{-1} \mathbf{A}_{2}^{T} \\
& \boldsymbol{\Sigma}_{2} \preceq I-\mathbf{A}_{1} \boldsymbol{\Sigma}_{1}^{-1} \mathbf{A}_{1}^{T} \\
& \mathbf{A}_{1}^{T}\left(\mathbf{H}_{22} \mathbf{Q}_{2}^{*} \mathbf{H}_{22}^{T}\right)^{-1} \mathbf{H}_{21}-\mathbf{H}_{11}=0 \\
& \mathbf{A}_{2}^{T}\left(\mathbf{H}_{11} \mathbf{Q}_{1}^{*} \mathbf{H}_{11}^{T}\right)^{-1} \mathbf{H}_{12}-\mathbf{H}_{22}=0,
\end{aligned}
$$

the sum capacity of $\operatorname{GXC}\left(\mathbf{H}_{11}, \mathbf{H}_{21}, \mathbf{H}_{12}, \mathbf{H}_{22}, P_{1}, P_{2}\right)$ is achieved by using the TIN scheme with cross messages only, and is given by

$$
\begin{aligned}
C_{\text {sum }}^{X}= & R_{T I N}^{X}\left(\mathbf{Q}_{1}^{*}, \mathbf{Q}_{2}^{*}\right) \\
= & \max _{\mathbf{Q}_{i} \in \mathcal{Q}_{i}, i \in\{1,2\}} \frac{1}{2} \log \mid I+\mathbf{H}_{21} \mathbf{Q}_{1} \mathbf{H}_{21}^{T} \\
& \quad \times\left(I+\mathbf{H}_{22} \mathbf{Q}_{2} \mathbf{H}_{22}^{T}\right)^{-1} \mid \\
& +\frac{1}{2} \log \left|I+\mathbf{H}_{12} \mathbf{Q}_{2} \mathbf{H}_{12}^{T}\left(I+\mathbf{H}_{11} \mathbf{Q}_{1} \mathbf{H}_{11}^{T}\right)^{-1}\right| .
\end{aligned}
$$

Proof: Apply Theorem 1 to the dual channel $\mathrm{GXC}\left(\mathbf{H}_{21}\right.$, $\left.\mathbf{H}_{11}, \mathbf{H}_{22}, \mathbf{H}_{12}, P_{1}, P_{2}\right)$ and use Remark 2 .

\section{Symmetric MISO AND SIMO GaUSSIAN XCS}

Now, we state the results for the symmetric MISO and SIMO Gaussian XCs. The main difference between the MISO and MIMO cases is in proving $R_{T I N}^{G A-X}\left(\mathbf{Q}_{1}^{*}, \mathbf{Q}_{2}^{*}\right)=$ $R_{T I N}^{X}\left(\mathbf{Q}_{1}^{*}, \mathbf{Q}_{2}^{*}\right)$. However, given that the genie-aided outer bound $R_{T I N}^{G A-X}\left(\mathbf{Q}_{1}, \mathbf{Q}_{2}\right)$ in Theorem 1 is the same as the bound in [2], this part remains the same as in [2, Theorem 2] and [2, Theorem 3]. Therefore, the proofs are not included.

\section{A. Symmetric MISO Gaussian XC}

A symmetric MISO Gaussian XC can be simplified to the following standard form [2] [4, Section 4.1]:

$$
\begin{aligned}
& y_{1}=\boldsymbol{d}^{T} \boldsymbol{x}_{1}+h \boldsymbol{c}^{T} \boldsymbol{x}_{2}+z_{1} \\
& y_{2}=h \boldsymbol{c}^{T} \boldsymbol{x}_{1}+\boldsymbol{d}^{T} \boldsymbol{x}_{2}+z_{2},
\end{aligned}
$$

where $\boldsymbol{d}=[\cos \theta \sin \theta]^{T}, \theta \in(0,(\pi / 2)), \boldsymbol{c}=\left[\begin{array}{ll}1 & 0\end{array}\right]^{T}$. Note that $\left(\mathbf{Q}_{1}^{*}, \mathbf{Q}_{2}^{*}\right)$ are unit rank matrices [5] in the MISO case and not full rank as required in Theorems 1 and 2 for the MIMO case. Here, we need to find sufficient conditions on $h$ for the TIN scheme to achieve sum capacity in the noisy interference regime. Following an approach similar to [2, Theorem 2], we get the following result.

Theorem 3: The sum capacity of the MISO Gaussian XC $\operatorname{GXC}\left(\boldsymbol{d}^{T}, h \boldsymbol{c}^{T}, h \boldsymbol{c}^{T}, \boldsymbol{d}^{T}, P, P\right)$ is given by

$$
C_{\text {sum }}^{X}= \begin{cases}\log \left[1+\frac{h^{2} P \cos ^{2} \theta}{1+P}+h^{2} P \sin ^{2} \theta\right], & h \geq h_{0}(\theta, P) \\ \log \left[1+\frac{P \cos ^{2} \theta}{1+h^{2} P}+P \sin ^{2} \theta\right], & h \leq h_{1}(\theta, P)\end{cases}
$$

where $h_{0}(\theta, P)$ and $h_{1}(\theta, P)$ are the positive solutions to the (29) and (30), respectively.

$$
\begin{aligned}
\left(\frac{1}{h_{0}^{2}}-\sin ^{2} \theta\right) & =\left(\frac{\cos \theta}{1+P}-\frac{1}{h_{0}}\right)_{+}^{2} \\
\left(h_{1}^{2}-\sin ^{2} \theta\right) & =\left(\frac{\cos \theta}{1+h^{2} P}-\frac{1}{h_{1}}\right)_{+}^{2} .
\end{aligned}
$$

Equation (30) specifies the condition under which sum capacity is achieved using the TIN scheme with direct messages only. Equation (29), which is obtained using the dual channel, specifies the condition under which sum capacity is achieved using the TIN scheme with cross messages only.

\section{B. Symmetric SIMO Gaussian XC}

A symmetric SIMO Gaussian XC can be simplified to the following standard form:

$$
\begin{aligned}
& \boldsymbol{y}_{1}=\boldsymbol{d} x_{1}+h \boldsymbol{c} x_{2}+\boldsymbol{z}_{1} \\
& \boldsymbol{y}_{2}=h \boldsymbol{c} x_{1}+\boldsymbol{d} x_{2}+\boldsymbol{z}_{2},
\end{aligned}
$$

where $\boldsymbol{d}=\left[\begin{array}{cc}\cos \theta & \sin \theta\end{array}\right]^{T}, \theta \in(0,(\pi / 2)), \boldsymbol{c}=\left[\begin{array}{ll}1 & 0\end{array}\right]^{T}$. Following an approach similar to [2, Theorem 3], we get this result.

Theorem 4: The sum capacity of the SIMO Gaussian XC $\operatorname{GXC}(\boldsymbol{d}, h \boldsymbol{c}, h \boldsymbol{c}, \boldsymbol{d}, P, P)$ is also given by (28) where $h_{0}(\theta, P)$, and $h_{1}(\theta, P)$ are the positive solutions to the equations (29) and (30), respectively. Under these conditions, the SIMO XC has the same sum capacity as the MISO XC. 


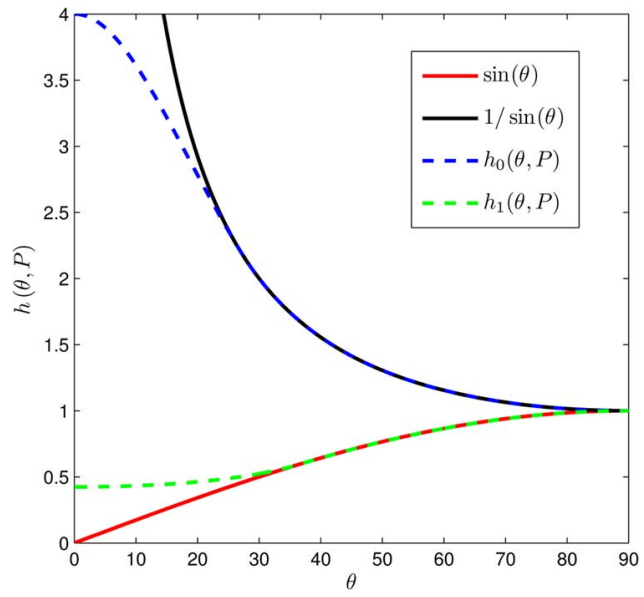

Fig. 2. Lower and upper bounds on $h$ for MISO and SIMO Gaussian symmetric XC with $P=0 \mathrm{~dB}$

\section{Numerical Results}

The noisy interference regime for the MISO and SIMO Gaussian XCs is illustrated in Fig. 2. If $h$ is greater than $h_{0}(\theta, P)$, or lesser than $h_{1}(\theta, P)$, the sum capacity is achieved using the TIN scheme. This noisy interference regime comprises a larger set of channels when compared to the MIMO IC result, which has only the $h_{1}(\theta, P)$ bound. The bounds for SISO XC, a special case of MISO XC with $\theta=0$, are: $\left|h\left(1+h^{2} P\right)\right|<$ $0.5,|(1+P) / h|<0.5$, and were derived in [3, Theorem 6.1]. As $P \rightarrow \infty, h_{0}(\theta, P)$ and $h_{1}(\theta, P)$ curves approach $1 / \sin (\theta)$ and $\sin (\theta)$ respectively.

\section{Symmetric Diagonal MiMO Gaussian XC}

In this section, we consider the case when $\mathbf{H}_{11}, \mathbf{H}_{12}, \mathbf{H}_{21}$, and $\mathbf{H}_{22}$ are $n \times n$ diagonal resulting in $n$ parallel SISO Gaussian XCs. For simplicity, we will focus on the case where each SISO channel is symmetric. Further, let $P$ be the total transmit power constraint for each transmitter in the MIMO XC. If each of the $n$ parallel XCs is in the noisy interference regime for SISO XCs, then the sum capacity of the diagonal MIMO XC is achieved by treating interference as noise in each channel and by optimally allocating power between the $n$ channels. We now determine the optimal power allocation that achieves sum capacity in this noisy interference regime.

For each of the $n$ parallel SISO XCs, either the cross links could be weak or the direct links could be weak. Let $h_{i}$ denote the channel coefficient corresponding the weak cross (or direct) links and let the direct (or cross) links be 1 . Since each channel is in the noisy interference regime, we have $2 h_{i}\left(1+h_{i}^{2} P\right) \leq 1$ for each $i$. Let $\alpha_{i} P$ be the power allocated by transmitter 1 to the $i$ th parallel $X$ channel, and $\beta_{i} P$ be the power allocated by transmitter 2 to the $i$ th parallel XC. For a given choice of $\alpha_{i}$ 's and $\beta_{i}$ 's, the sum capacity of the symmetric diagonal MIMO Gaussian $\mathrm{XC}$ is

$$
C\left(\alpha_{1}, \cdots, \alpha_{n}, \beta_{1}, \cdots, \beta_{n}\right)=\sum_{i=1}^{n} C_{X}\left(h_{i}, \alpha_{i}, \beta_{i}\right)
$$

where $C_{X}\left(h_{i}, \alpha_{i}, \beta_{i}\right)$ is the sum capacity of the $i$ th parallel SISO $\mathrm{XC}$ in the noisy interference regime given by

$C_{X}\left(h_{i}, \alpha_{i}, \beta_{i}\right)=\frac{1}{2} \log \left(1+\frac{\alpha_{i} P}{1+h_{i}^{2} \beta_{i} P}\right)$

$$
+\frac{1}{2} \log \left(1+\frac{\beta_{i} P}{1+h_{i}^{2} \alpha_{i} P}\right) .
$$

The sum capacity of the symmetric diagonal MIMO Gaussian $\mathrm{XC}$ is obtained by maximizing the above sum capacity in (32) over all $\alpha_{i}$ 's and $\beta_{i}$ 's subject to $\sum_{i=1}^{n} \alpha_{i} \leq 1, \sum_{i=1}^{n} \beta_{i} \leq 1$, $\alpha_{i} \geq 0$, and $\beta_{i} \geq 0$.

Theorem 5: The sum capacity of the symmetric diagonal MIMO Gaussian XC is achieved by choosing $\alpha_{i}=\beta_{i}$ for each $i$ and

$$
\alpha_{i}=\left[\frac{\sqrt{1+4 \nu P h_{i}^{2}\left(h_{i}^{2}+1\right)}-\left(2 h_{i}^{2}+1\right)}{2 h_{i}^{2}\left(h_{i}^{2}+1\right) P}\right]^{+}
$$

with $\nu>0$ chosen such that $\sum_{i=1}^{n} \alpha_{i}=1$.

Proof: First, observe that $C_{X}\left(h_{i}, \alpha_{i}, \beta_{i}\right)=C_{X}\left(h_{i}, \beta_{i}\right.$, $\left.\alpha_{i}\right)$. Furthermore, the component of the gradient of $C_{X}\left(h_{i}, \alpha_{i}\right.$, $\beta_{i}$ ) in the direction normal to the $\alpha_{i}=\beta_{i}$ line can be shown to be non-negative for $\alpha_{i} \geq \beta_{i}$ and $2 h_{i}^{2}\left(1+h_{i}^{2} P\right) \leq 1$. Therefore, $\alpha_{i}=\beta_{i}$ is optimal. Now, we need to maximize

$$
\sum_{i=1}^{n} C_{X}\left(h_{i}, \alpha_{i}, \alpha_{i}\right)=\sum_{i=1}^{n} \log \left(1+\frac{\alpha_{i} P}{1+h_{i}^{2} \alpha_{i} P}\right) .
$$

Since $C_{X}\left(h_{i}, \alpha_{i}, \alpha_{i}\right)$ is increasing in $\alpha_{i}$, the optimal $\alpha_{i}$ 's in (34) can be obtained using the Lagrangian dual and the KarushKuhn-Tucker conditions.

Remark 3: As expected, more power is allocated to a channel with smaller $h_{i}$, i.e., lesser interference. However, the solution is different from standard water-filling since the first term in (34) also depends on $h_{i}$ and does not correspond to a constant "water-level".

\section{CONCLUSIONS}

Two noisy interference regimes were determined for the MIMO Gaussian XC corresponding to the two TIN schemes: one with direct messages only, and the other with cross messages only. Thus, the noisy interference regime of the MIMO $\mathrm{XC}$ comprises a larger subset of channel conditions than the noisy interference regime of MIMO IC. This larger regime was illustrated using numerical results for MISO and SIMO Gaussian XCs. The symmetric diagonal MIMO Gaussian XC was also studied and an optimal power allocation determined in the noisy interference regime.

\section{REFERENCES}

[1] V. S. Annapureddy and V. V. Veeravalli, "Gaussian interference networks: Sum capacity in the low interference regime and new outer bounds on the capacity region," IEEE Trans. Inf. Theory, vol. 55, no. 7, pp. 3032-3050, Jul. 2009.

[2] V. S. Annapureddy and V. V. Veeravalli, "Sum capacity of MIMO interference channels in the low interference regime," IEEE Trans. Inf. Theory, vol. 57, no. 5, pp. 2565-2581, May 2011.

[3] C. Huang, V. R. Cadambe, and S. A. Jafar, "Interference alignment and the generalized degrees of freedom of the X channel," IEEE Trans. Inf. Theory, vol. 58, no. 8, pp. 5130-5150, Aug. 2011.

[4] X. Shang and B. Chen, "Two user Gaussian interference channels: An information theoretic point of view," Found. Trends Commun. Inf. Theory, vol. 10 , no. 3 , pp. 247-378, Dec. 2013.

[5] X. Shang, B. Chen, and H. V. Poor, "Multi-user MISO interference channels with single-user detection: Optimality of beamforming and the achievable rate region," IEEE Trans. Inf. Theory, vol. 57, no. 7, pp. 4255-4264, Jul. 2011. 